\title{
Patient care for postamputation pain and the complexity of therapies: living experiences
}

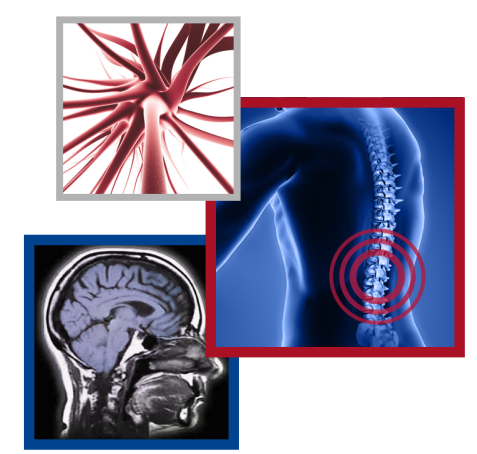

\author{
Marjorie Flahaut ${ }^{\ddagger} 1$, Nicolas L Laurent ${ }^{\ddagger}, 1$, Murielle Michetti ${ }^{1}$, Nathalie Hirt-Burri ${ }^{1}$, Winnie \\ Jensen², Romulus Lontis², Lee A Applegate*,‡,1 \& Wassim Raffoul ${ }^{\ddagger}, 1$ \\ ${ }^{1}$ Department of Plastic, Reconstructive \& Hand Surgery, Unit of Regenerative Therapy, University Hospital of Lausanne, 1066 \\ Epalinges, Switzerland \\ ${ }^{2}$ Department of Health Science \& Technology, Center for Sensory-Motor Interaction, Aalborg University, 9000 Aalborg, Denmark \\ *Author for correspondence: Lee.Laurent-Applegate@chuv.ch \\ $\ddagger$ Authors contributed equally
}

\section{Practice points}

- Phantom limb pain (PLP) is very common among amputees, with up to $85 \%$ of concerned people beginning to feel intense neuropathic pain in the missing limb rapidly after the amputation time.

- Pharmacological treatments are commonly used to treat PLP in a first line of management, but their efficacy remains limited.

- Most of the patients are refractory to pharmacological treatments and are often in favor of testing other noninvasive and nonpharmacological approaches.

- Complementary and alternative medicine therapies can be helpful for the reduction of PLP.

- The very high medication administered in first and second or sometimes third intentions often alters significantly the physical and mental state of the patient.

- As chronic pain affects the whole person (body, mind and spirit), the complementary and alternative medicine therapies in combination with more traditional therapies can allow the patients to take care of themselves at multiple levels and to actively participate in their own healing process and wellbeing.

- Each patient could eventually receive a combination of conventional and alternative therapies according to their own pain.

- Progress in understanding the pathophysiological mechanisms will also allow to develop new procedures to precisely identify this type of pain and thus implement complementary interventions or treatments.

- It is crucial to continue this type of research that requires multidisciplinary teams to effectively follow overall patient care and to define a 'personalized therapeutic plan' to better help these patients with extreme suffering.

Aim: Limb amputation traumatically alters body image. Sensations rapidly prevail that the limb is still present and $85 \%$ of patients portray phantom limb pain. Throughout the testimonies of amputated patients with intense phantom limb pain, we show the difficulty in treating this chronic pain with current pharmacological and nonpharmacological therapies. Patients \& methods: We qualitatively analyzed the therapeutic choices of five amputees, the effectiveness of the treatments chosen and the impact on patients' quality-of-life. Results \& conclusion: In general, patients who are refractory to pharmacological treatments are in favor of trying alternative therapies. It is therefore crucial to design a combined and personalized therapeutic plan under the coordination of a multidisciplinary team for the wellbeing of the patient.

First draft submitted: 25 May 2018; Accepted for publication: 16 August 2018; Published online: 3 September 2018

Keywords: chronic pain • complementary and alternative medicine therapies $\bullet$ neuropathic pain $\bullet$ phantom limb pain • pharmacological therapies $\bullet$ postamputation pain $\bullet$ rehabilitation $\bullet$ therapy

Chronic pain is defined to be persistent for a longer period than expected and that can be refractory to classical treatments [1]. Chronic low back pain, headache, fibromyalgia, neuropathic pain and central pain syndromes are common clinical syndromes in patients suffering from chronic pain. The high prevalence of patients with chronic pain such as neuropathic (7-10\%) is due to the modest efficiency of the commonly used medication including 
opioids and nonsteroidal anti-inflammatory drugs. The effects of these drugs remain largely subjective, as one patient may respond and another may not $[2,3]$. The lack of efficiency surrounding chronic pain mechanisms is also not yet clearly defined and this indeed prevents the identification of specific target molecules. People affected by chronic pain have highly altered quality-of-life in consequence to sleep disturbances, anxiety and depression and this is a growing problem in our society as demonstrated by the global epidemic of opioid abuse [3-5]. Patients are often so desperate that they go from doctor to doctor trying to find a new treatment solution adapted to their pain. Moreover, they try to relieve their pain by using alternative therapies and this has been reported for many years [6].

Phantom limb pain (PLP) is part of neuropathic pain that is precisely defined as a pain caused by a lesion or disease of the somatosensory system and associated with the perception of touch, pressure, pain, temperature, position, movement and vibration [3]. PLP commonly arises from an injury to the peripheral nervous system. Lesions of the peripheral nerves or the CNS, which are typically caused by a brachial-plexus avulsion or paraplegia, can also cause PLP [7]. The misrepresented sensations that are behind this peripheral nerve damage likely originates from inappropriate signaling from the peripheral neuron to its second-order targets, with multifactorial errors in both transduction and transmission pathways of the pain signal $[8,9]$.

As previously mentioned, the peripheral nerve system has been shown to be implicated in the development of PLP $[10,11]$. This observation has been controversial, as studies have indicated that additional mechanisms involving the CNS must exist [12-14]. Robust studies indicate that the CNS is involved in the development of the PLP with an evident structural reorganization of the spinal cord as well as the primary somatosensory cortex after peripheral nerve damage like in the case of an amputation [15-18]. Flor et al. contributed largely to show that PLP is attributed to many mechanisms, including spinal plasticity [7] or cortical remapping [19,20]. Neuroimaging studies have been readily useful for patients with peripheral neuropathies to highlight the multiple changes in activity and functional connections that can occur in the regions of the brain that are involved in pain processing and pain modulation $[9,21,22]$. From the site of amputation, receptors called nociceptors send signals to the spinal cord (transduction) and then to the brain (transmission) where the pain is treated (perception) (Figure 1). Central mechanisms underlying the evolution from a phantom sensation to phantom pain are localized within either the brain or the spinal cord. At the brain level, those mechanisms consist principally in the reorganization of the somatosensory cortex by invasion of the region of the brain (homunculus diagram, Figure 1), representing the missing limb by neighboring areas of the somatosensory and motor cortex [23,24]. The spinal cord, which is the connection site between the injured site (amputation) and the brain, will also be reorganized and resensitized by loss of the afferent signal from the peripheral nerves. Peripheral mechanisms are triggered by the primary lesion of the nerves at the site of injury that are no longer able to transmit a correct afferent nerve input to the spinal cord and stimulate subsequent formation of a hypersensitive neuroma [25]. Nevertheless, a recent review of neuroimaging studies has shown that evidence for a link between cortical reorganization and pain in amputees or in patients with spinal cord injury is limited and controversial. Better standardized studies are, therefore, essential in the future to better understand this relationship [26].

PLP is very common among amputees, with up to $85 \%$ of concerned people who begin to feel pain in the missing limb rapidly after the amputation $[27,28]$. It has been evaluated that PLP can appear within 1 week after amputation in the majority of patients, but half of the patients will experience PLP during the first $24 \mathrm{~h}$ after the operation [14]. This high heterogeneity in the PLP mechanisms as previously described has the consequence of multiple treatment possibilities [25]. PLP is considered as an important healthcare problem due to the growing number of patients with amputations that subsequently develop other pathologies such as obesity, cardiovascular disease and sleep disorder among others. A study published in 2008 showed that there were about 1.6 million people with limb loss in the USA and this number was estimated to more than double to approximately 3.6 million in 2050 [29]. Jensen et al. have evaluated the average pain intensity according to the Visual Analog Scale (VAS) to 5.3 out of 10 in a study. The pain has been shown to increase over time for $50 \%$ of patients with the other $50 \%$ of patients reporting no change in pain intensity over time. The patients described their pain as shooting, shocking, burning, electrical-like sensations, cramping or dysesthesia like itching or tingling, as well as nonpainful sensations, several days per month $[3,30]$. The reason why some patients develop PLP after amputation and some patients do not is still largely unknown. Several factors seem to be associated with the development of this chronic pain that includes peripheral, central and psychological factors interacting with each other [14,27].

Pharmacological treatments are commonly used to treat PLP in a first line of management, but although many treatment modalities such as botulinum neurotoxins, nonsteroidal anti-inflammatory drugs, opioids, tricyclic antidepressants (inhibition of serotonin-norepinephrine uptake blockade, $N$-methyl-D-aspartate receptor antagonism 


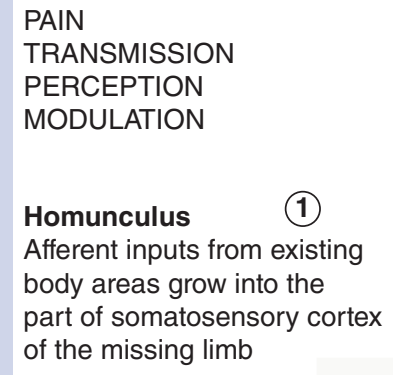

Homunculus

Afferent inputs from existing body areas grow into the part of somatosensory cortex of the missing $\operatorname{limb}$

\section{Brain}

Modulation of cognitive behavior therapy motor cortex stimulation

Secretion of endogenous opioids

Spinal cord

Modulation of neuroplasticity and inhibition of interneurons in the spinal cord

Dorsal horn ganglion

\section{Nociceptor}

Modulation of nociceptor activation following tissue injury and inflammation
TREATMENTS
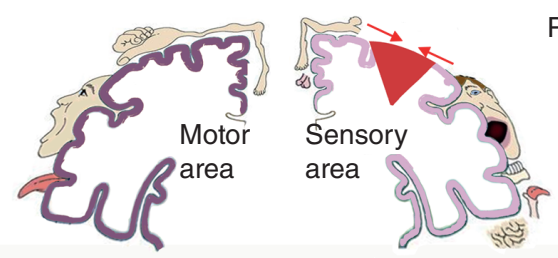

PHARMACOLOGIC

NON-

PHARMACOLOGIC

\section{Sensorimotor training}

Opioids MOR-NRI Cannabinoids

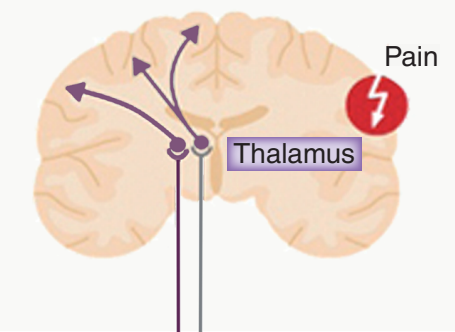

Opioids MOR-NRI Antidepressants Anticonvulsants Cannabinoids (channel blockers) NMDA blockers

Local anesthetics Antidepressants Anticonvulsants Cannabinoids Anti-inflammatory (NSAID)
Mental imagery

Augmented reality therapy Virtual reality therapy

Mirror box therapy

\section{Music}

Vibration therapy Hypnotic imagery Magnetic stimulation Electroshock ECT

TENS

Acupuncture

Use of prothesis
Biofeedback
Repositioning of residual limb
Massage of residual limb
Acupunture

\section{TENS}

Muscular electrostimulation Acupuncture

Figure 1. Schematic representation of the pain-signaling pathway. Diagram showing the sites of action of different categories of pharmacologic and nonpharmacologic treatments, as well as their potential in the modulation of pain between the peripheral nervous system and CNS (brain and spinal cord). From the site of injury, such as an amputation, nociceptors send signals to the spinal cord (transduction) and then to the brain (transmission) through the ascending pathways where the pain is treated (perception). Pain signal is then modulated through the descending pathways. On the left hand side of the figures are mentioned the different parts of the human body that are implicated in the pain pathway from the site of injury to the homunculus in the brain via the spinal cord. On the right hand side of the diagram, the different options among pharmacological and nonpharmacological treatments are listed as well as their known sites of action along the signaling pain pathway.

NSAID: Non-steroidal anti-inflammatory drug; TENS: Transcutaneous electrical nerve stimulation.

and sodium channel blockade), anticonvulsants, $N$-methyl-D-aspartate receptor antagonists, local anesthetics or calcitonin have been proposed, their efficacy remains limited [14,31-36]. Already in 1980, it has been postulated that as many as 43 treatments were used to manage PLP and since almost 40 years, the list of treatment possibilities such as drugs, surgery and alternative therapies has not finished to grow [37].

In the search of new emerging pharmacological agents indicated in this therapeutic field, cannabis-based drugs have been shown to be effective pharmacologic agents for treating pain through many randomized and controlled clinical trials [38]. Despite an intense controversial debate, medical marijuana is now commonly used in clinics for patients with chronic pain around the world [39]. Medical benefits of cannabis-derived compounds, like marijuana have been demonstrated. However, the health risks from the use of marijuana, as many other drugs still exist and clinical physicians, as well as patients should be well informed and aware about the practical implication of medical marijuana both at the scientific and legal levels [40]. The pathway and discussions toward medical legalization of 


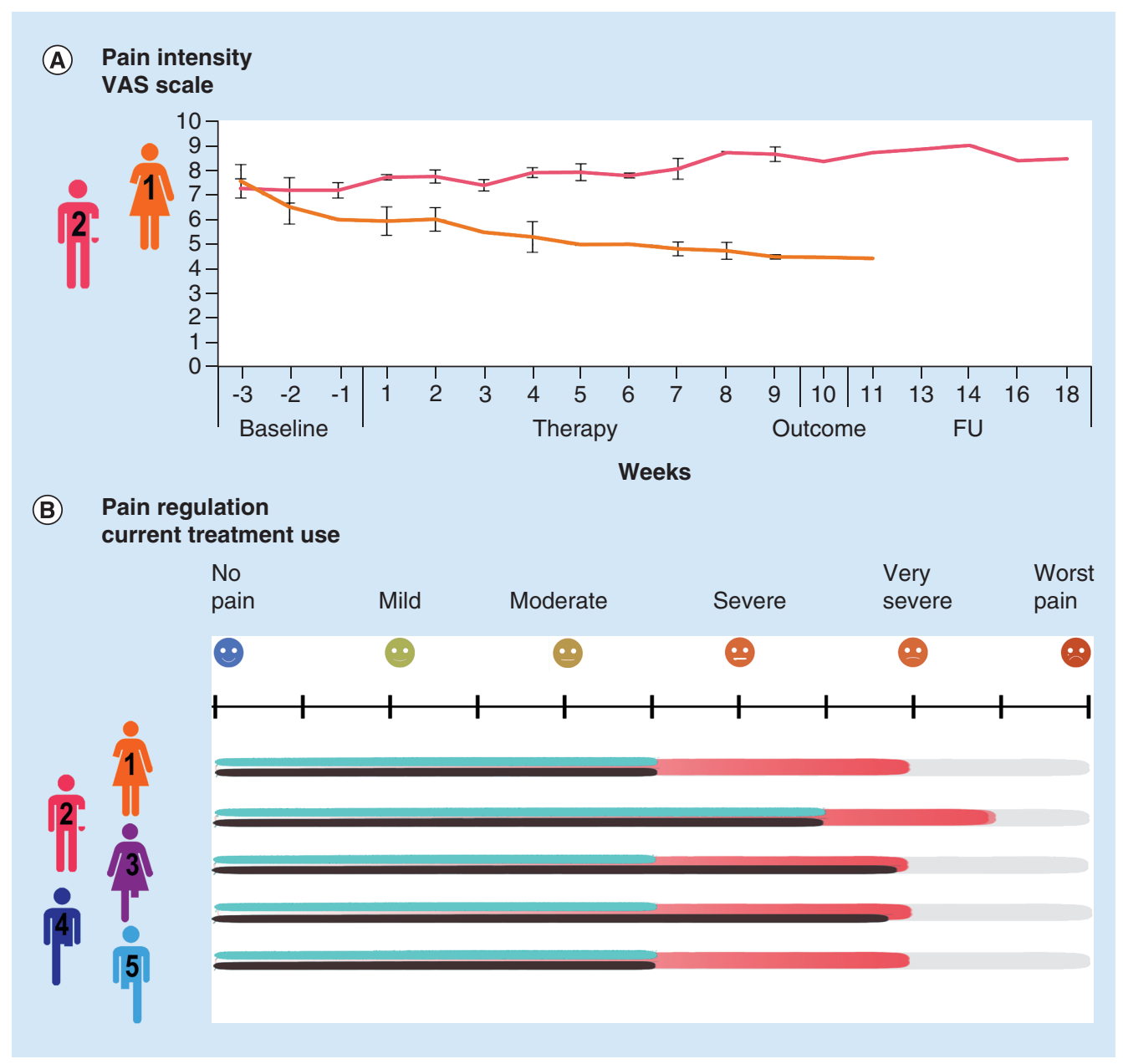

Figure 2. Evaluation of pain intensity. Evaluation of pain intensity (visual analog scale) $24 \mathrm{~h}$ before the beginning of therapy sessions by electrical stimulation was done for the two patients who participated in the study under the EPIONE protocol. The 3-month study period was divided into a 3-week baseline phase (control measures), a 9-week therapy phase with electrical stimulation sessions three-times per week, a 1-week period for evaluation of the outcome and, finally, a follow-up period to assess the long-term effect of the therapy. At each session during the successive phases of the protocol, patients 1 and 2 were asked to score their highest experienced pain level before the session using the software platform (A). The intensity of pain (from severe pain to no pain, red bar) was estimated for each patient according to their respective testimonies, as well as the impact of pharmacological (black bars) or nonpharmacological treatments (blue bars) on their pain relief (B).

VAS: Visual analog scale.

marijuana are important when we know that patients receiving medical marijuana reported that they were able to reduce or stop opioids that still lead to death by overdoses [41].

For nonpharmacological treatments, several approaches are used as invasive or noninvasive treatments for managing pain [42]. Complementary and alternative medicine therapies (CAM) can be helpful for the reduction of PLP and include many methods such as direct intraneural stimulation, acupuncture, massage of the residual limb, mirror box therapy, biofeedback, transcutaneous electrical nerve stimulation, treatment by electrochocs, repetitive transcranial magnetic stimulation, virtual reality (VR)/augmented reality (AR), mental imagery, hypnotic imagery, vibration therapy, musical therapy, as well as use of prosthesis.

Several studies have shown promise for hypnotherapy but only based on individual cases. There is an obvious need for controlled and randomized trials to confirm the efficacy of this therapy for PLP reduction. As was mentioned earlier, psychological, peripheral and central neural factors should help to explain the mechanism of phantom pain that still remain uncertain. Hypnosis is one of the therapy options that can change the perception of pain by directly targeting the psychological and emotional components of the pain [43]. 
Pharmacologic treatments

Non-pharmacologic treatments

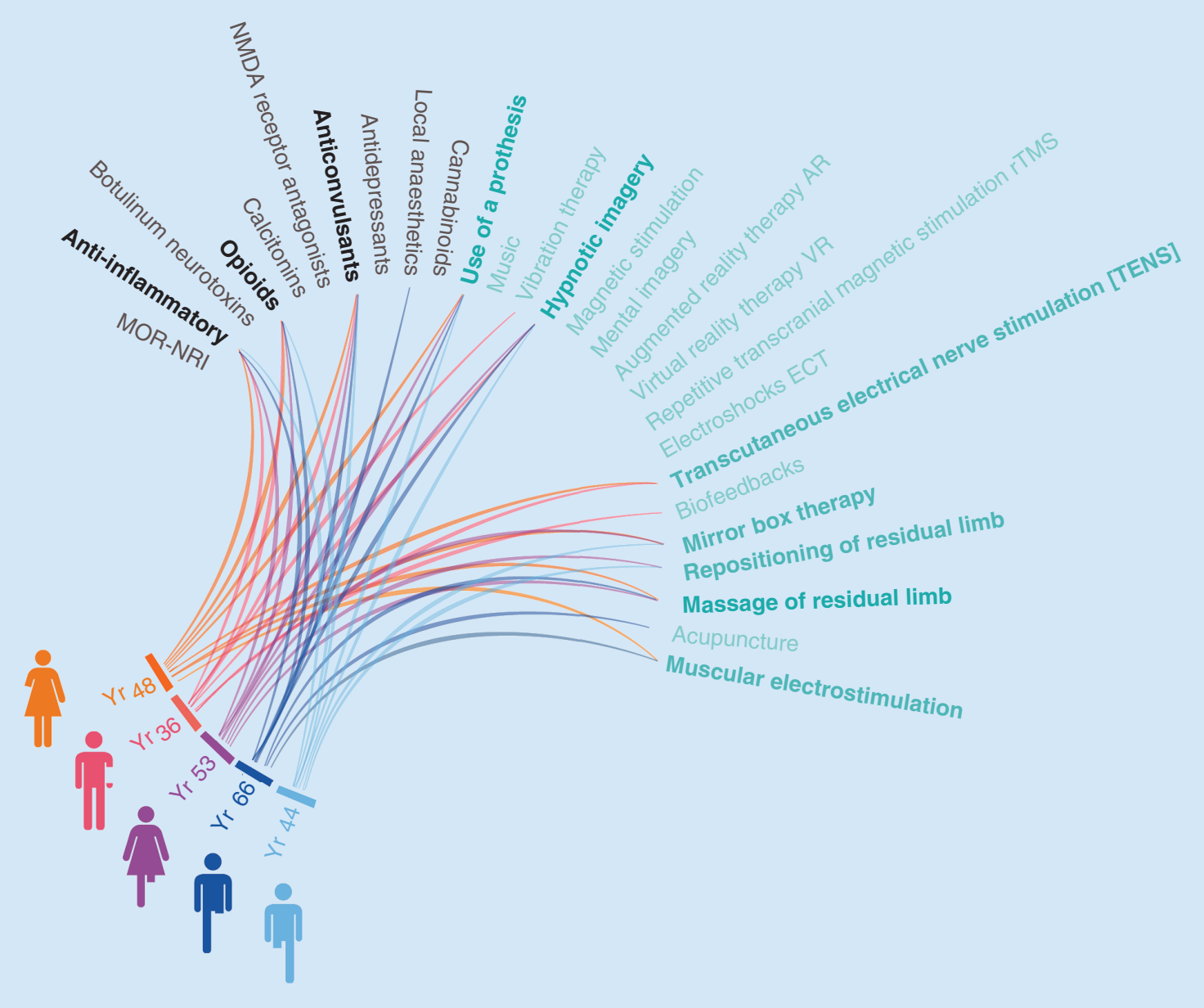

Figure 3. Therapeutic choices of five patients with neuropathic/phantom pain among the currently available pharmacologic and nonpharmacologic treatments. The diagram illustrates the vast therapy options that could be proposed to patients suffering from phantom limb pain. Among those different therapeutic strategies, the five patients have been treated according to conventional pharmacologic agents, such as anti-inflammatory, opioids and anticonvulsants drugs (black letters on the left). Currently available nonpharmacologic treatments (blue letters) to combine with the pharmacologic options are listed on the right and therapeutic options commonly tested by the five patients are highlighted.

Another alternative therapy is emerging today with acupuncture. More and more patients are currently trying this treatment due to the low incidence of side effects in comparison to pharmacologic treatments or invasive approaches. It has been shown that the mechanism of action of acupuncture on chronic pain is related to both the CNS and peripheral nervous system by brain neural activity modulation and endogenous factor release, respectively [44]. Despite the increasing use of acupuncture worldwide in clinics, its efficiency for treating chronic pain is still uncertain due to the poor study design. More recently, promising approaches directly derived from the mirror therapy are the treatments based on AR/VR that are offering a more immersive experience for the amputees. However, a literature review of these new techniques showed that higher quality studies are needed to demonstrate their efficiency [45].

By focusing on the testimony of five different patients from our institution who suffer from intense and sometimes untolerable phantom pain, we provide an overview of their therapeutic choices among the wide range of existing therapeutic options that are constantly in development in order to challenge the status-quo in the PLP treatment. We also discuss the importance of the therapeutic management by the medical staff in a multidisciplinary team. 


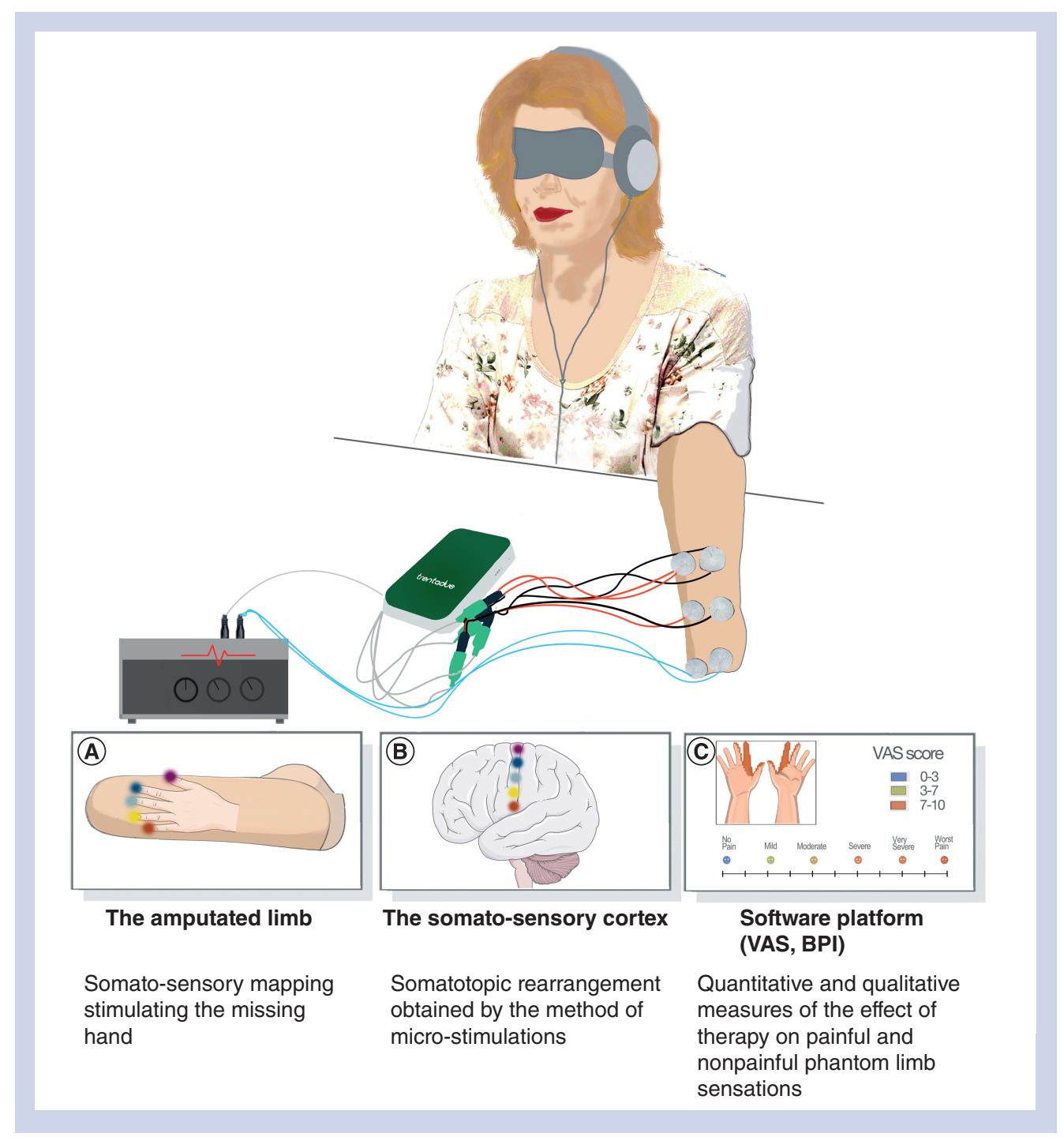

Figure 4. Study end points for a natural sensory feedback by transcutaneous electrical stimulations in amputee patients. One part of the European EPIONE project is based on a noninvasive approach of transcutaneous electrical stimulations in order to create nonpainful sensations to the amputated patient suffering from phantom limb pain (A) and therefore alleviate the pain intensity by restoring neuroplastic changes in the cortex (B). A software platform was developed in order to quantitatively and qualitatively follow the effect of the therapy on the painful and nonpainful phantom limb sensations, as well as the psychological state of the patients through multiple questionnaires (C). VAS: Visual analog scale.

\section{Patients \& methods}

The study has described experiences of five patients with known conditions. The study has used the COREQ checklist for this purpose. Patient interviews were carried out as part of a Federal Maturity project of a high school student and were at the hospital and in two medical offices. Two of five patients (one male, one female) were participating in parallel in a European clinical study (EPIONE, NCT02569918), on the hospital site and accepted to participate in the interviews in accordance with the ethical standards of the regional committee on human experimentation and with the Helsinki Declaration of 2013. The male student followed three other amputees with neuropathic pain during their consultations in medical offices with their respective treating male physicians. Patients were asked to participate in interviews in a prior consultation several weeks before and were asked specifically if a student could be present. Prior to the study, three patient relationships to the interviewer were that of the primary care physician to patient, and for two patients that of surgeon to patient. All of the interview sessions were conducted 


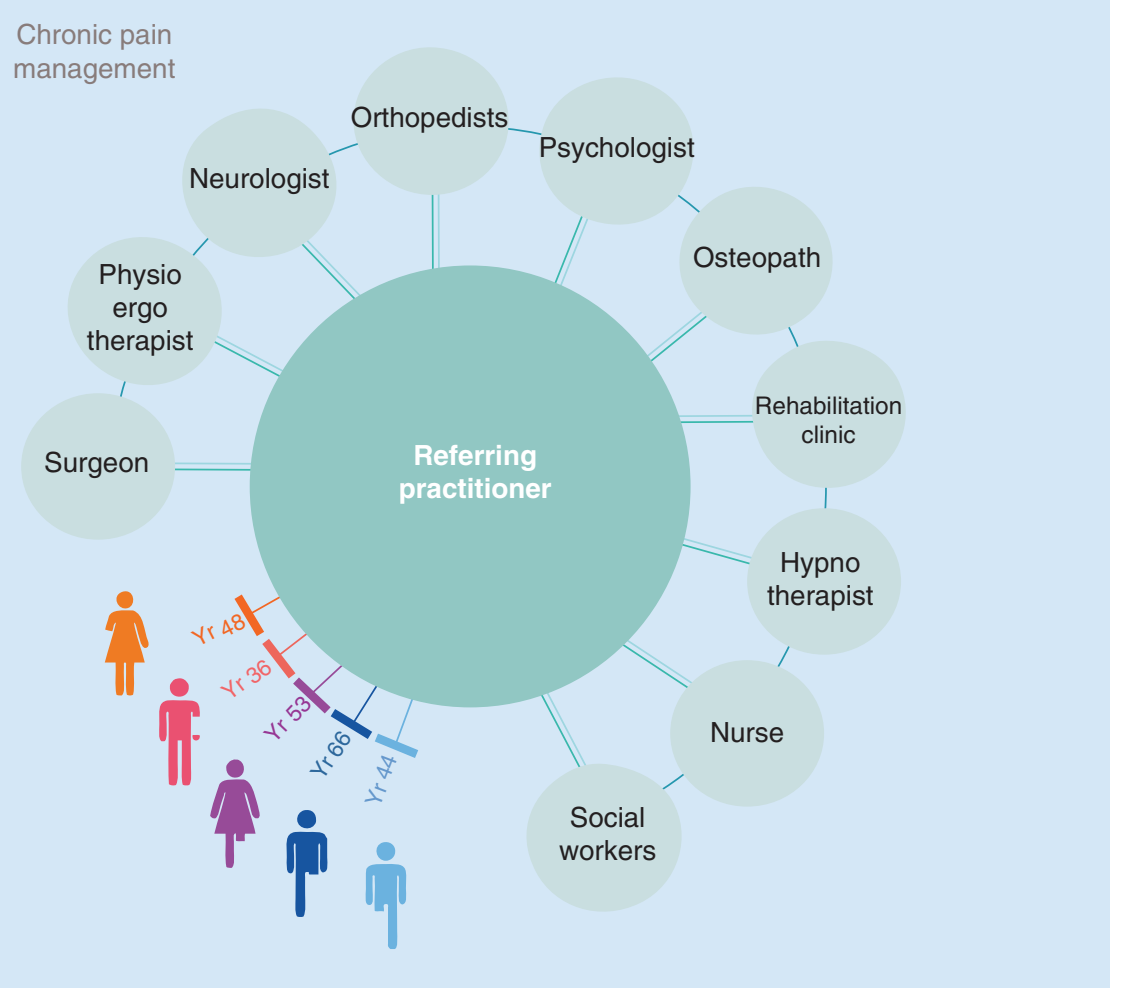

Figure 5. Chronic pain management. To assure that the patient suffering from chronic pain has an optimal and personalized therapeutic plan, a specialist's network is needed with the patient in the center. At the center of the figure and directly linked to the patient is the referring specialist who is responsible to maintain the main therapeutic plan, as well as the discussion network in the multidisciplinary specialists team potentially involved in the pain management whatever the moment or the evolution of the pain.

with the patient consent in presence of a doctor with the specific purpose of the interview to acquire knowledge from their personal therapeutic pathway.

Five patients (one upper limb- [female], three lower limb-amputees [two males, one female] and one patient [male] with a plexus brachial lesion) were evaluated for their participation to extensive interviews about the therapeutic experiences they have chosen for managing/treating their PLP to provide data for review. The interviews of the full five patients were regulated under the State Ethics regulations, which allow five case reports.

A patient with an upper limb-amputation (female) and a patient with a plexus brachial lesion (male) had also been included in a European multicenter study involving eight different countries implicated at clinical, industrial and academic levels (http://project-epione.eu/) as they met the inclusion criteria. Each patient provided their consent to participate in this aforementioned study. By applying electrical stimulation at the surface of the stump during a period of 3 months for therapy sessions, the hypothesis was to help relieve the pain by training the brain that the missing limb is still there. The interviews for the two patients were based on questionnaires for pain evaluation such as VAS [46] and Brief Pain Inventory [47] standards in accordance with the ethical regulation. They are classified as semistructured interviews according to qualitative medical research in order to collect appropriate information about the patient [48]. The main points discussed during the interviews with the patients concerned the causes of the disease (accident, disease), the history of amputation and the existence of postoperative rehabilitation period (when, where, how long). The patients were asked in more details about the appearance of PLP (when, how, intensity) and which type of treatment has been organized by the doctors and by the patient for the management of pain. The impact of the different treatments that have been applied was also evaluated by each patient as well as their long-term efficiency.

The three other patients who did not meet the inclusion criteria participated in the intensive interview only and were audio recorded during a 30-min period. Interviews were discussed with each patient after summaries were written for the report. 


\section{Results}

Following the testimonies of the interviewed patients, we analyzed their therapeutic choices and effectiveness of these treatments on their phantom limb/neuropathic pain taking all the information together in order to assess the complexity of therapies available to put forth an optimal care pathway.

\section{Patient \#1}

Patient \#1 was a 48-year-old woman with a distal transradial amputation of the right hand. PLP appeared a few days after the amputation and was described as a warm sensation of itching and/or tingling. She was able to partially imagine her phantom hand with some fingers, and particularly the middle finger, which she was unable to unfold. She had not used a functional prosthesis regularly, but had experienced use of prosthesis in the past during rehabilitation periods without any success nor in relation to operating the prosthesis, nor in relation to pain reduction. She took analgesics daily and opioid pain medication such as Tramal ${ }^{\text {TM }}$ (Grünenthal Pharma AG, Switzerland). Moreover, the patient tried mirror therapy, which failed to reduce pain. She reported every day an intense PLP (7-8/10 on the VAS) that was stronger in the evening and at night, therefore disrupting her regular sleep patterns. She accepted to participate in the EPIONE noninvasive study. During 3 months, she came threetimes per week to the hospital to receive the therapy based on surface electrical stimulations for $1 \mathrm{~h}$ per session. During the last month, therapy was applied with the introduction of hand prosthesis. Unfortunately, the patient did not feel like there had been a significant change in pain intensity, frequency or type; however, she reported having an improved sensation in the fingers. As such, she felt her phantom limb as more vivid, more clearly defined, and she described this as a positive outcome for herself. The change in the pain intensity as shown in Figure $2 \mathrm{~A}$ was significant with a VAS score of 7.5 at the beginning of the first phase and 4.5 at the end of the therapy phase. At the end of the study, she was able to open her phantom hand almost completely and to feel all the fingers in contrast to the beginning of the study. Thereafter, she tried muscular electrostimulation without any success. Unfortunately, several months after the end of the study without continued therapy her phantom fingers fell back to their initial position and sensation (Pers. Comm. from E. D'Anna).

\section{Patient \#2}

Patient \#2 was a 36-year-old man who suffered from a brachial plexus injury of the left shoulder. Since the accident, the patient felt an intense burning pain at the level of his left hand. His forearm was also partially paralyzed and sometimes he experienced severe pain in his elbow and shoulder. The neuropathic pain was characterized mainly as electrical shocks or stress points in addition to intense burning sensation. Since his accident, his quality-of-life had been considerably impaired not only due to the high level of neuropathic pain but also by the use of high doses of medication. He received the maximum daily dose of the anti-epileptic drug, Lyrica ${ }^{T M}$ (Pfizer PFE Switzerland $\mathrm{GmbH}$, Zürich, Switzerland) that is commonly used for neuropathic pain. Before Lyrica, he had taken other medication that left him comatose. He was then asked by his physician to participate in the EPIONE noninvasive study. Unfortunately, his pain was found to increase during treatment (Figure 2A) and he started to feel electric shocks more frequently. At the end of the follow-up, which represented 4 weeks after end of therapy by electrical stimulation, the frequency of electrical shocks was restored to the same level that he experienced before entering the study. In addition, his pain remained very high. After discussion with an occupational therapist, he decided to begin vibration therapy and he has become highly interested and motivated to use neurofeedback therapy as well as hypnotherapy.

\section{Patient \#3}

Patient \#3 was a 53-year-old woman who has a left leg amputation. The patient was not able to identify the exact time when the phantom pain appeared due to high doses of prescribed anti-inflammatory drugs, morphine and anticonvulsants, which altered her feeling of pain. Moreover, she was unable to distinguish the phantom pain from the postoperative pain of the stump. The patient frequently experienced noninvasive therapies such as mirror therapy and hypnosis, where the latter was considered effective in reducing pain. The patient described an acute localized pain without warning or progression in the intensity. She was able to say precisely that she felt intense pain within a toe, under the soles of the feet or under the heel. She says: "The only thing I can say is that the pain comes in a violent and unexpected way. We can be discussing now and in a second, I am bent in two, crying. You are not warned: you are shopping, and you are suddenly screaming in the middle of the shop, without any alert when it would be happening. It is simply there and it is too late. We cannot do anything. It is precisely localized. 
I can say exactly where I hurt." The different drugs have had no effect on the intensity of the phantom pain and the patient definitively stopped them. Each patient can be very different and therefore pain intensity or sensation is subjective. This particular patient does not feel itching or burning but she has felt her foot as if it is there again and completely blocked in the prosthesis. It was never comfortable. She describes her foot like a cramp mixed with a burn.

\section{Patient \#4}

Patient \#4 was a 66-year-old man who lost his left leg. Like for many patients experiencing an amputation, the phantom pain appeared only a few days after the amputation intervention. Immediately after surgery, the patient received various drugs such as morphine to relieve postoperative pain. He quickly decided to stop morphine because he was completely against this kind of medication. When the pain was attributed to neuropathic pain by the doctors, antiepileptic drugs such as Lyrica were administered to the patient, but he again refused to take them, thinking that it would have no effect on his pain. The phantom pain was really agonizing and highly impaired the quality of sleep with only $1 \mathrm{~h}$ of sleep per night for long periods of time. The patient described his pain either like a tingling or like an intense pain along his leg as if a truck was rolling over him or as if his leg was clenched in a vice. He spent a long period at the hospital and many therapies had been proposed to him but none really showed effectiveness. In contrast, these therapies generally accentuated the intensity of the pain, which greatly discouraged the patient. During the rehabilitation period, the patient tried different strategies to relieve the pain, especially muscular electrostimulation without any success. He also tried acupuncture and experienced more intense pain. The only therapy he tested which had a positive effect on his pain was hypnosis therapy, but unfortunately that was not reimbursed by the insurance company and he was forced to stop treatment due to the economic impact. At one point, he expressed a desperate message: "either you do something or I will hang myself." He was then directed to a pain center where he first received intense medication that gave no improvement. Thereafter, the medical staff proposed spinal cord stimulation that consisted of the implantation of a stimulator under the skin in the back, which would be able to transmit low electrical current to the spinal cord. This method is known to treat chronic pain [49]. The patient refused to test this technology arguing that a friend had already tried it with no benefit. Since this point, he regularly had called the 'faiseurs de secret', magnetizers and psychotherapists who had finally but transiently relieved the pain. He has tried to find the best solution for him and to live with this pain in peace.

\section{Patient \#5}

Patient \#5 was a 44-year-old male patient with a right leg amputation. The phantom pain was felt 3-days postamputation and appeared first like a tingling but the pain became more and more intense the following days and weeks. He had the terrible feeling that someone was twisting his leg, and at other times, he felt that his foot was compressed in a ski boot. The patient began to receive increasing doses of antianalgesic medication to prevent the pain from reaching an intolerable threshold and also to provide the patient with a better quality of sleep. He tried rapidly to combine hypnosis and mirror therapy. Pain clearly decreased during the hypnosis sessions but was still present after. In general, the patient felt less pain with time by receiving hypnosis as well as mirror therapy. For mirror therapy, the patient received a treatment according to three phases: First, he was asked to look at pictures of limbs corresponding to his missing one, and second he was asked to visualize the missing limb in the correct position. Third, he tried mirror therapy sessions during which he could see the reflexion of his intact limb in the mirror and so he could make his brain believe that his missing limb was still there. High concentration from the patient was required for this technique whose efficiency is highly subjective. However, for this patient mirror therapy has been shown to be effective in reducing pain, but it did not make it disappear. The pain he felt at the beginning like a twist or a compression became a lighter feeling with a foot in a normal position and wrapped in a cotton bed. Lately, the pain level is 'liveable' but the patient is still under the control of high medication. The patient had also expressed that it was the help of his son taking his first steps while he was at home for rehabilitation that helps him live with his pain. These moments of everyday life have given him the force to move forward and to combat and relativize his PLP.

\section{Discussion}

The interview experience with five different patients suffering from phantom/neuropathic pain shows us that patients are often trying several treatment approaches without any real success in alleviating their pain. Under most 
circumstances, people with neuropathic pain suffer a major degradation of their quality-of-life and consequently of their daily activities in relation with sleeping, mobility, working and personal interactions.

After a first-line pharmacological management of the pain, patients chose therapies not only in agreement with their doctor but also in relation to their confidence in one method or another. The panel of pharmacological and nonpharmacological treatments that are offered in general to these patients is very large and each treatment is able to act at different levels along the pain-signaling pathway (Figures $1 \& 3$ ). There is obviously not a single or combined consensus treatment. The patients discussed in this paper suffer greatly from their pain, and are often subjected to high doses of medication that can render them nonfunctional to live normally, or even be in a comatose state. Very often, the alternative and complementary therapies remain combined with pharmacological treatments. Compliance of the patient is very important and depends highly in the confidence in a technique and trust for the doctor. These treatments require regularity (number of visits, duration of visits, personalized exercises) and will demonstrate little effectiveness if they are not applied precisely and, importantly, if not supervised by a competent therapist. Patients with neuropathic pain should have regular clinical follow-up as the response to treatment can be subjective to the mental/psychological state of the patient.

International groups work to develop guidelines for the pharmacologic management of neuropathic pain according to results obtained from clinical studies, but it seems very difficult to decide a consensus-based pharmacologic treatment for patients that are supported for neuropathic pain, as well as for other chronic pain [50]. A high number of studies described in the review of Cliff Richardson in 2017 have tried to demonstrate the potential of pharmacological compounds against PLP following an amputation or a nerve injury with either effectiveness on a short-term period, or no effect or controversial effects [51]. Unfortunately, no single treatment seems to emerge even now for these desperate patients. In general, combined pharmacological therapies are proposed to the patients with the goal to reduce each drug independently and therefore cause fewer side effects. We had also seen that, in general, treatments administered for the five patients interviewed in our study had only a little effect on the pain intensity (Figure 2B). Sometimes, patients mentioned that pain was even increasing during different treatment regimens [Pers. Comm. from Patient 4].

The use of CAM including acupuncture, hypnosis, deep-breathing exercise, massage therapy, meditation, naturopathy, yoga, mind-body system, dietary interventions and fasting, as well as herbal medicine has been increasing since more than 10 years [52-57]. As chronic pain affects the whole person (body, mind and spirit), the CAM therapies in combination with more traditional therapies could allow the patient to take care of himself at multiple levels and to actively participate in his own healing process and wellbeing. These techniques seem to be effective in the management of chronic pain but much remains to do to understand their mechanism of action and their true effectiveness in the self-management of pain by the patient [44,58].

Promising technological approaches include treatments based on AR/VR that are offering a more immersive experience for the amputees. However, a literature review of these new techniques showed that higher quality studies are needed to demonstrate their efficiency [45]. Through a clinical study combining mirror therapy and motor imagery including 14 upper-limb amputees who were refractory to conventional treatments for many years, study investigators were able to demonstrate a $50 \%$ pain reduction [59]. Nevertheless, more research in the future is needed to improve and demonstrate the potential benefit of these technologies.

We have shown with the two patients that had responded to the questionnaires that the therapy effect could be lesion-dependent, as the two patients suffering from intense neuropathic pain did not respond in the same way. Effectively one patient was subject to neuropathic pain following an amputation while the second patient suffered a brachial plexus injury. However, this difference could simply be due to the many criteria that exist between two individuals. A recent and innovative study showed that it is possible to induce referred sensations to the phantom hand of amputees by a transcutaneous electrical nerve stimulation approach. This technique combined with the control of bidirectional hand prosthesis allowed the patient to perform different functional tasks by a feedback control of the sensations, but no impact on the pain was illustrated in this context of this case study [60].

Invasive treatments, such as surgical resection of neuroma, ablation of spinal cord dorsal root, thalamotomy or sympathectomy could be proposed for patients who are completely refractory to pharmacological treatments, but they only provide short-term improvement in PLP and are highly associated with severe complications and risk of relapse. Other surgical procedures, like the direct spinal cord stimulation or the intracranial neurostimulation have also been proposed but showed only a short-term relief of pain [61]. A few years ago, it has been shown that intraneural stimulation under the control of prosthesis has been also efficient for restoring the sense of touch in an amputee [62]. Following these previous experiments, invasive treatment based on the development of new 
technology for implantable neurostimulatory electrodes [63] that could be directly inserted in the nerves of the stump for a direct intraneural electrical stimulation [64] has been developed. The EPIONE clinical trial included a noninvasive intervention, which consisted in the application of surface electrical stimulations in the stump region on referred sensation areas and therefore creating natural sensations in the missing or paralyzed limb according to a specific stimulation program [60]. The treatment used in this specific study was based on recent evidence that PLP may be related to neuroplastic changes in the cortex and that these changes may be modulated by providing sensory input to the residual limb or amputation zone [65]. Figure 4 illustrates the EPIONE study as a whole.

Developing new technological and nonpharmacological solutions that will noninvasively or invasively induce natural, meaningful sensations to the amputee represent a challenge and need to be optimized to be integrated into routine choices for patients.

Overall, it was of interest to evaluate the evolution of alternative approaches in pain management and to show the variety of therapies that had been and that could be proposed to a patient according to his pain characteristics and his compliance.

\section{Conclusion}

It is important to continue multidisciplinary team research approaches to effectively follow overall patient care and to define a 'personalized therapeutic plan' to better help these patients with extreme suffering (Figure 5). By working together, varied approaches can be added to the therapeutic choices to optimize patient care. In any case, the patient would benefit from a patient-center approach with a referring therapist in whom there is full trust. The therapist will coordinate the medical network around the patient in order to offer a solid and personalized therapeutic plan in agreement with individual beliefs.

\section{Acknowledgements}

The extensive interviews were accomplished under the scope of a Federal Maturity project by author NL Laurent. The content is solely the responsibility of the authors and does not necessarily represent the official views of the European Commission.

Financial \& competing interests disclosure

The study has been funded, in part, by the European Union FP7-HEALTH-2013INNOVATION (project no. 602547 'EPIONE') and for the most part by the University Hospital of Lausanne, Switzerland. The authors have no other relevant affiliations or financial involvement with any organization or entity with a financial interest in or financial conflict with the subject matter or materials discussed in the manuscript apart from those disclosed.

No writing assistance was utilized in the production of this manuscript.

Ethical conduct of research

Two of five patients were participating in a European clinical study (EPIONE, NCT02569918), on the hospital site and accepted to participate in the interviews in accordance with the ethical standards of the regional committee on human experimentation and with the Helsinki Declaration of 2013. Signed informed consents were obtained in accordance with the ethical statement. Three amputated patients with neuropathic pain have been interviewed during their consultations in medical offices with their respective treating physicians. All the interviews sessions were conducted with the patient consent in presence of a doctor.

\section{Open access}

This work is licensed under the Attribution-NonCommercial-NoDerivatives 4.0 Unported License. To view a copy of this license, visit http://creativecommons.org/licenses/by-nc-nd/4.0/

\section{References}

1. Treede RD, Rief W, Barke A et al. A classification of chronic pain for ICD-11. Pain 156(6), 1003-1007 (2015).

2. Cohen SP, Mao J. Neuropathic pain: mechanisms and their clinical implications. BMJ 348, f7656 (2014).

3. Colloca L, Ludman T, Bouhassira D et al. Neuropathic pain. Nat. Rev. Dis. Primers 3, 17002 (2017).

4. Jensen MP, Chodroff MJ, Dworkin RH. The impact of neuropathic pain on health-related quality of life: review and implications. Neurology 68(15), 1178-1182 (2007).

5. Kolodny A, Courtwright DT, Hwang CS et al. The prescription opioid and heroin crisis: a public health approach to an epidemic of addiction. Annu. Rev. Publ. Health 36, 559-574 (2015).

6. Ashburn MA, Staats PS. Management of chronic pain. Lancet 353(9167), 1865-1869 (1999). 
7. Flor H. Phantom-limb pain: characteristics, causes, and treatment. Lancet Neurol. 1(3), 182-189 (2002).

8. Meacham K, Shepherd A, Mohapatra DP, Haroutounian S. Neuropathic pain: central vs. peripheral mechanisms. Curr. Pain Headache R. 21(6), 28 (2017).

9. Sandkuhler J. Models and mechanisms of hyperalgesia and allodynia. Physiol. Rev. 89(2), 707-758 (2009).

10. Chabal C, Jacobson L, Russell LC, Burchiel KJ. Pain responses to perineuromal injection of normal saline, gallamine, and lidocaine in humans. Pain 36(3), 321-325 (1989).

11. Devor M, Janig W, Michaelis M. Modulation of activity in dorsal root ganglion neurons by sympathetic activation in nerve-injured rats. J. Neurophysiol. 71(1), 38-47 (1994).

12. Harwood DD, Hanumanthu S, Stoudemire A. Pathophysiology and management of phantom limb pain. Gen. Hosp. Psychiat. 14(2), 107-118 (1992).

13. Kooijman CM, Dijkstra PU, Geertzen JH, Elzinga A, Van Der Schans CP. Phantom pain and phantom sensations in upper limb amputees: an epidemiological study. Pain 87(1), 33-41 (2000).

14. Weeks SR, Anderson-Barnes VC, Tsao JW. Phantom limb pain: theories and therapies. Neurologist 16(5), 277-286 (2010).

15. Baron R. Mechanisms of disease: neuropathic pain - a clinical perspective. Nat. Clin. Pract. Neurol. 2(2), $95-106$ (2006).

16. Flor H, Elbert T, Muhlnickel W, Pantev C, Wienbruch C, Taub E. Cortical reorganization and phantom phenomena in congenital and traumatic upper-extremity amputees. Exp. Brain Res. 119(2), 205-212 (1998).

17. Florence SL, Kaas JH. Large-scale reorganization at multiple levels of the somatosensory pathway follows therapeutic amputation of the hand in monkeys. J. Neurosci. 15(12), 8083-8095 (1995).

18. Woolf CJ, Shortland P, Coggeshall RE. Peripheral nerve injury triggers central sprouting of myelinated afferents. Nature 355(6355), 75-78 (1992).

19. Flor $\mathrm{H}$, Elbert T, Knecht $\mathrm{S}$ et al. Phantom-limb pain as a perceptual correlate of cortical reorganization following arm amputation. Nature 375(6531), 482-484 (1995).

20. Flor H, Nikolajsen L, Staehelin Jensen T. Phantom limb pain: a case of maladaptive CNS plasticity? Nat. Rev. Neurosci. 7(11), 873-881 (2006).

21. Moisset X, Bouhassira D. Brain imaging of neuropathic pain. NeuroImage 37(Suppl. 1), S80-S88 (2007).

22. Seifert F, Maihofner C. Central mechanisms of experimental and chronic neuropathic pain: findings from functional imaging studies. Cell. Mol. Life Sci. 66(3), 375-390 (2009).

23. Foell J, Bekrater-Bodmann R, Flor H, Cole J. Phantom limb pain after lower limb trauma: origins and treatments. Int. J. Low. Extr. Wound 10(4), 224-235 (2011).

24. Ramachandran VS, Hirstein W. The perception of phantom limbs. The D. O. Hebb lecture. Brain 121(Pt 9), 1603-1630 (1998).

25. Subedi B, Grossberg GT. Phantom limb pain: mechanisms and treatment approaches. Pain Res. Treat. 2011, 864605 (2011).

26. Jutzeler CR, Curt A, Kramer JL. Relationship between chronic pain and brain reorganization after deafferentation: a systematic review of functional MRI findings. Neuroimage Clin. 9, 599-606 (2015).

27. Luo Y, Anderson TA. Phantom limb pain: a review. Int. Anesthesiol. Clin. 54(2), 121-139 (2016).

28. Sherman RA, Sherman CJ. Prevalence and characteristics of chronic phantom limb pain among American veterans. Results of a trial survey. Am. J. Phys. Med. 62(5), 227-238 (1983).

29. Ziegler-Graham K, MacKenzie EJ, Ephraim PL, Travison TG, Brookmeyer R. Estimating the prevalence of limb loss in the United States: 2005 to 2050. Arch. Phys. Med. Rehab. 89(3), 422-429 (2008).

30. Jensen TS, Krebs B, Nielsen J, Rasmussen P. Immediate and long-term phantom limb pain in amputees: incidence, clinical characteristics and relationship to pre-amputation limb pain. Pain 21(3), 267-278 (1985).

31. Buvanendran A, Kroin JS. Early use of memantine for neuropathic pain. Anesth. Analg. 107(4), 1093-1094 (2008).

32. Flor H. Maladaptive plasticity, memory for pain and phantom limb pain: review and suggestions for new therapies. Expert Rev. Neurother. 8(5), 809-818 (2008).

33. Hanley MA, Ehde DM, Campbell KM, Osborn B, Smith DG. Self-reported treatments used for lower-limb phantom pain: descriptive findings. Arch. Phys. Med. Rehab. 87(2), 270-277 (2006).

34. Mittal SO, Safarpour D, Jabbari B. Botulinum toxin treatment of neuropathic pain. Semin. Neurol. 36(1), 73-83 (2016).

35. Verdu B, Decosterd I, Buclin T, Stiefel F, Berney A. Antidepressants for the treatment of chronic pain. Drugs 68(18), 2611-2632 (2008).

36. Wiffen PJ, McQuay HJ, Edwards JE, Moore RA. Gabapentin for acute and chronic pain. Cochrane DB Syst. Rev. (3), Cd005452 (2005).

37. Sherman RA. Published treatments of phantom limb pain. Am. J. Phys. Med. 59(5), 232-244 (1980).

38. Hill KP. Medical marijuana for treatment of chronic pain and other medical and psychiatric problems: a clinical review. JAMA 313(24), 2474-2483 (2015).

39. Bifulco M, Pisanti S. Medicinal use of cannabis in Europe. EMBO Rep. 16(2), 130-132 (2015). 
40. Wilsey B, Atkinson JH, Marcotte TD, Grant I. The medicinal cannabis treatment agreement: providing information to chronic pain patients through a written document. Clin. J. Pain 31(12), 1087-1096 (2015).

41. Haroutounian S, Ratz Y, Ginosar Y et al. The effect of medicinal cannabis on pain and quality-of-life outcomes in chronic pain: a prospective open-label study. Clin. J. Pain 32(12), 1036-1043 (2016).

42. Demir Y. Non-pharmacological therapies in pain management. In: Pain Management - Current Issues and Opinions. Gabor Racz (Ed.). InTech, Rijeka, Croatia, 485-502 (2012). www.intechopen.com/books/pain-management-current-issues-and-opinions/non-pharmacol ogicaltherapies-in-pain-management

43. Komendova I. Practical application of hypnosis in treatment of phantom limb pain. Ann. Psychol. (02), 56-66 (2015).

44. Chen L, Michalsen A. Management of chronic pain using complementary and integrative medicine. BMJ 357, j1284 (2017).

45. Dunn J, Yeo E, Moghaddampour P, Chau B, Humbert S. Virtual and augmented reality in the treatment of phantom limb pain: a literature review. NeuroRehabilitation 40(4), 595-601 (2017).

46. McCormack HM, Horne DJ, Sheather S. Clinical applications of visual analogue scales: a critical review. Psychol. Med. 18(4), 1007-1019 (1988).

47. Cleeland CS, Ryan KM. Pain assessment: global use of the Brief Pain Inventory. Ann. Acad. Med. 23(2), 129-138 (1994).

48. Britten N. Qualitative interviews in medical research. BMJ311(6999), 251-253 (1995).

49. Carter ML. Spinal cord stimulation in chronic pain: a review of the evidence. Anaesth. Intensive Care 32(1), 11-21 (2004).

50. O'Connor AB, Dworkin RH. Treatment of neuropathic pain: an overview of recent guidelines. Am. J. Med. 122(10 Suppl.), S22-S32 (2009).

51. Richardson C, Kulkarni J. A review of the management of phantom limb pain: challenges and solutions. J. Pain Res. 10, 1861-1870 (2017).

52. Griffin SC, Tsao JW. A mechanism-based classification of phantom limb pain. Pain 155(11), 2236-2242 (2014).

53. Berger MM, Davadant M, Marin C et al. Impact of a pain protocol including hypnosis in major burns. Burns 36(5), 639-646 (2010).

54. Batsford S, Ryan CG, Martin DJ. Non-pharmacological conservative therapy for phantom limb pain: a systematic review of randomized controlled trials. Physiother. Theor. Pr. 33(3), 173-183 (2017).

55. Bradbrook D. Acupuncture treatment of phantom limb pain and phantom limb sensation in amputees. Acupunct. Med. 22(2), 93-97 (2004).

56. Davies A. Acupuncture treatment of phantom limb pain and phantom limb sensation in a primary care setting. Acupunct. Med. 31(1), 101-104 (2013).

57. Trevelyan EG, Turner WA, Summerfield-Mann L, Robinson N. Acupuncture for the treatment of phantom limb syndrome in lower limb amputees: a randomised controlled feasibility study. Trials 17(1), 519 (2016).

58. Lee C, Crawford C, Swann S. Multimodal, integrative therapies for the self-management of chronic pain symptoms. Pain Med. 15(Suppl. 1), S76-S85 (2014).

59. Ortiz-Catalan M, Guethmundsdottir RA, Kristoffersen MB et al. Phantom motor execution facilitated by machine learning and augmented reality as treatment for phantom limb pain: a single group, clinical trial in patients with chronic intractable phantom limb pain. Lancet 388(10062), 2885-2894 (2016).

60. D’Anna E, Petrini FM, Artoni F et al. A somatotopic bidirectional hand prosthesis with transcutaneous electrical nerve stimulation based sensory feedback. Sci. Rep. 7(1), 10930 (2017).

61. Viswanathan A, Phan PC, Burton AW. Use of spinal cord stimulation in the treatment of phantom limb pain: case series and review of the literature. Pain Pract. 10(5), 479-484 (2010).

62. Raspopovic S, Capogrosso M, Petrini FM et al. Restoring natural sensory feedback in real-time bidirectional hand prostheses. Sci. Transl. Med. 6(222), 222ra219 (2014).

63. Stieglitz T, Boretius T, Navarro X et al. Development of a neurotechnological system for relieving phantom limb pain using transverse intrafascicular electrodes (TIME). Biomed. Tech. 57(6), 457-465 (2012).

64. Krahenbuhl SM, Cvancara P, Stieglitz T et al. Return of the cadaver: key role of anatomic dissection for plastic surgery resident training. Medicine 96(29), e7528 (2017).

65. Andoh J, Milde C, Tsao JW, Flor H. Cortical plasticity as a basis of phantom limb pain: fact or fiction? Neuroscience doi:https://doi.org/10.1016/j.neuroscience.2017.11.015. (2017) (Epub ahead of print). 
